\title{
An illustration of an in-vivo corneal response to a soft lens presoaked in a non-isotonic solution
}

\author{
A. J. KEMPSTER AND J. R. LARKE \\ From the Department of Ophthalmic Optics, University of Aston, Birmingham
}

SUMMARY The use of hydrophilic gel contact lenses as a carrier of a non-isotonic saline solution provokes an irreversible increase in corneal thickness in the in-vivo rabbit eye. Studies on humans confirm the nature of the animal response, and it is therefore suggested that particular care should be taken when patients prepare saline solutions.

Patients wearing soft hydrogel contact lenses may prepare their own saline storage solutions from distilled water and commercially available salt tablets. There is evidence to suggest that solutions prepared by patients are not always made up to the recommended strength (Boyd, 1975). Work previously reported by the present authors (Kempster et al., 1975) has shown an evoked corneal thickness change when incorrect preparation by a patient results in a hypertonic storage solution. Further work, now reported, suggests that changes in corneal thickness may also be incited from storage in hypotonic saline solutions.

Twelve Bausch and Lomb ' $N$ ' series Soflenses were presoaked for 24 hours, 6 in $0.7 \%$ saline and a further 6 in $0.9 \%$ saline. After autoclaving at $120^{\circ} \mathrm{C}$ for 20 minutes the lenses were placed on the eyes of 6 albino male New Zealand rabbits of approximately $1.5 \mathrm{~kg}$. The experimental eye was fitted with the lens presoaked in $0.7 \%$ saline, while the contralateral control eye was fitted with the lens soaked in $0.9 \%$ saline. A Haag-Streit pachometer, modified after Mishima and Hedbys (1968), was used to measure central corneal thickness. After 5 hours the lenses were removed and the animals observed for a further period of 12 days, after which time they were killed and the corneae subjected to histological examination.

The collected data are illustrated graphically in Fig. 1. It is apparent that there is a significant increase $(P=0.01)$ in the thickness of the corneae subjected to the wearing of the lenses presoaked in hypotonic saline. Similarly, the histological sections of the experimental and control corneae also show

Address for reprints: Dr J. R. Larke, Department of Ophthalmic Optics, University of Aston, Gosta Green, Birmingham B4 7ET

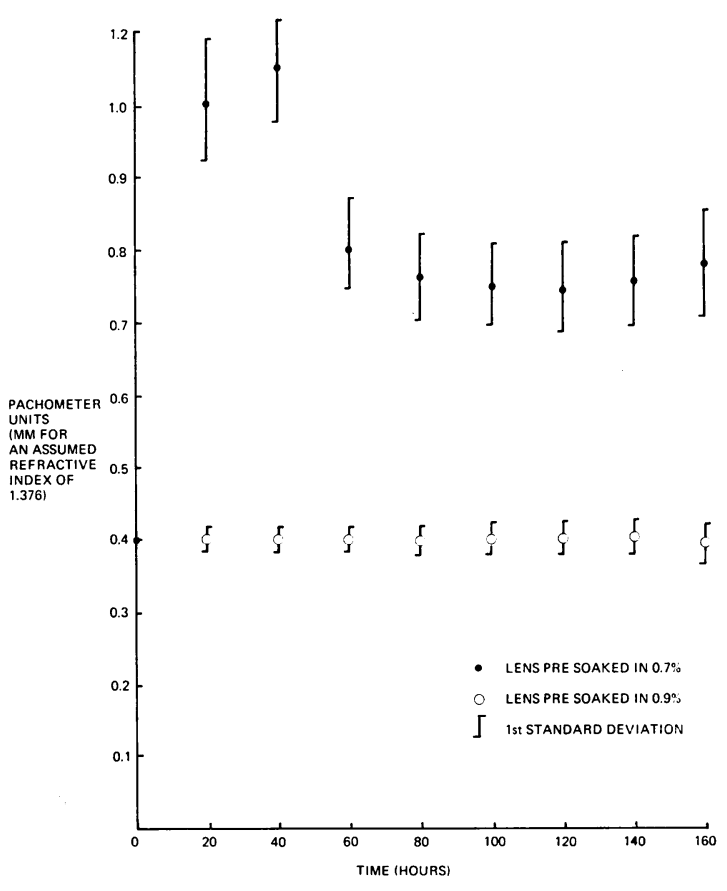

Fig. 1 Corneal thickness (rabbit)

marked differences in the corneal thickness (Figs. 2, $3,4)$.

Corneal swelling in the rabbits was in excess of that expected, and posed ethical problems when human experimentation was considered. It was decided to pursue limited human work on 6 volunteers, who were informed of the results of the animal work. They were limited to 2 hours' exposure and a corneal thickness change of no more than 2.5 pachometer units. The authors submitted themselves 


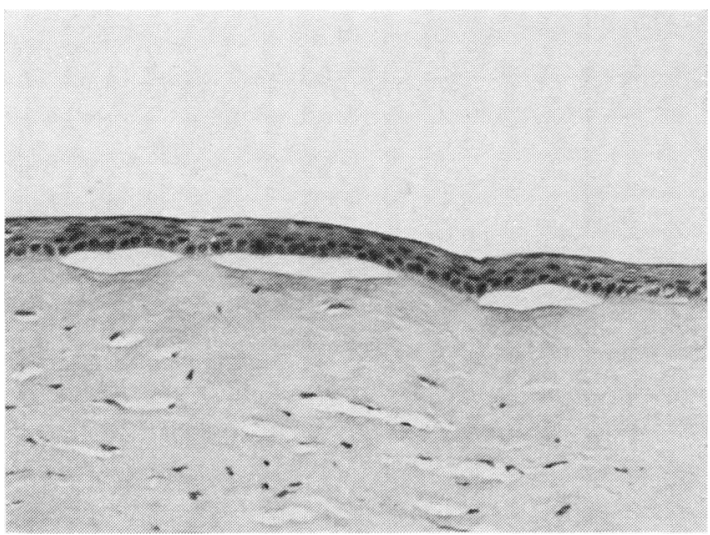

Fig. 2 General view of epithelium and stroma showing 'bullae' (fluid-filled vesicles) at the epithelial/stroma interface. Gross stromal disruption is also evident (Haematoxylin and eosin) to 4 hours of lens wear before beginning this study and participated as subjects in the 2-hour study. In addition to topographic corneal thickness measurement estimations of chloride ion levels in tear fluid were also made. A glass capillary tube was used for removal of 2 to $3 \mathrm{ml}$ of unstimulated tears from the 'tear lake', which were subjected to conventional electrochemical measurement for chloride ion evaluation.

The results of the human study are illustrated graphically in Figs. 5, 6, and 7. Fig. 5 shows a significantly $(P=0 \cdot 1)$ declining tear chloride ion level after 2 hours of lens wear. Comparison with Fig. 6 illustrates a statistically significant $(P=0.01)$ increase in corneal thickness for the same experimental subjects. It is apparent from Fig. 7, which illustrates the corneal thickness change for a peripheral region of the cornea, that the change in thickness is not evenly reflected in all regions of

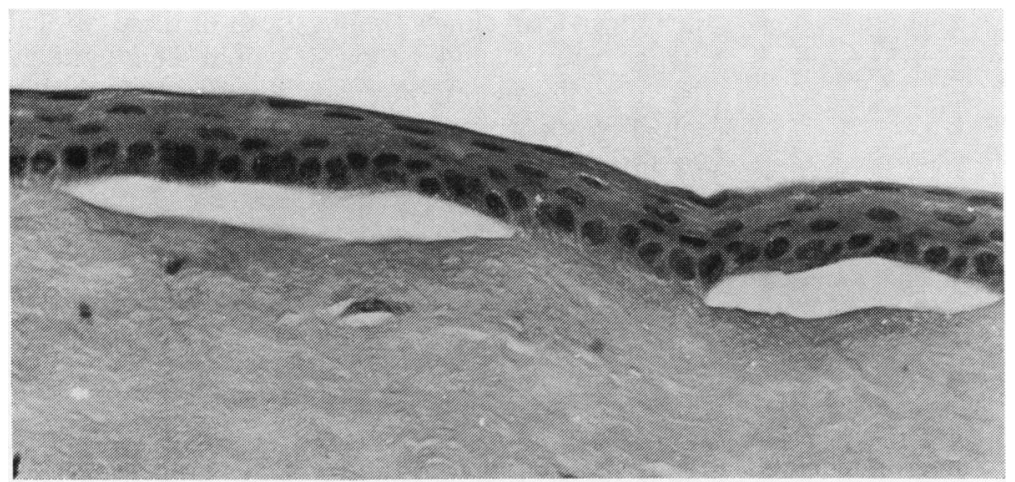

Fig. 3 'Bullae' under higher magnification. The appearance of the bullae is consistent with that seen by slit-lamp examination before killing the animal

Fig. 4 Grossly disturbed stroma with some evidence of stromal vesicles

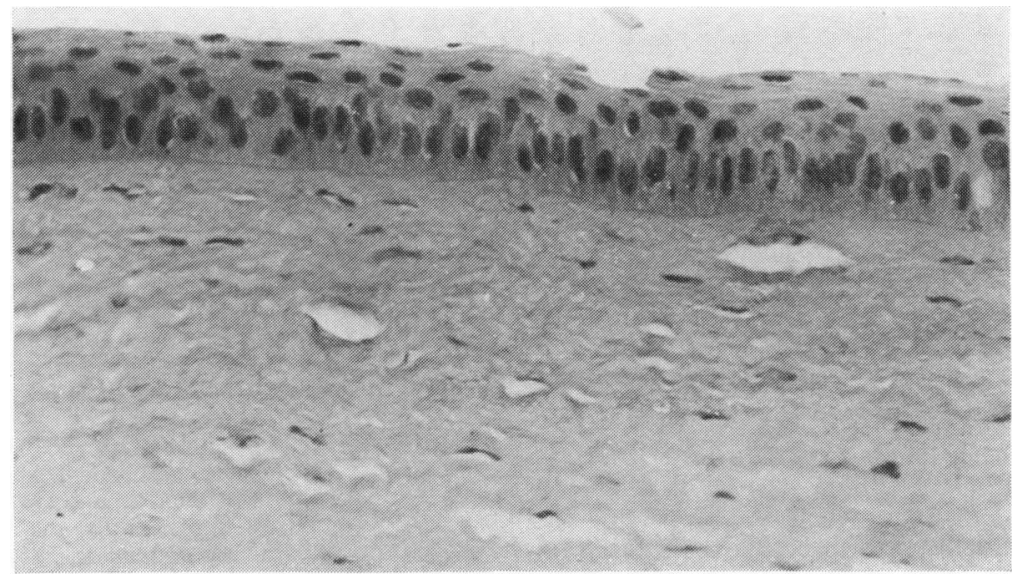




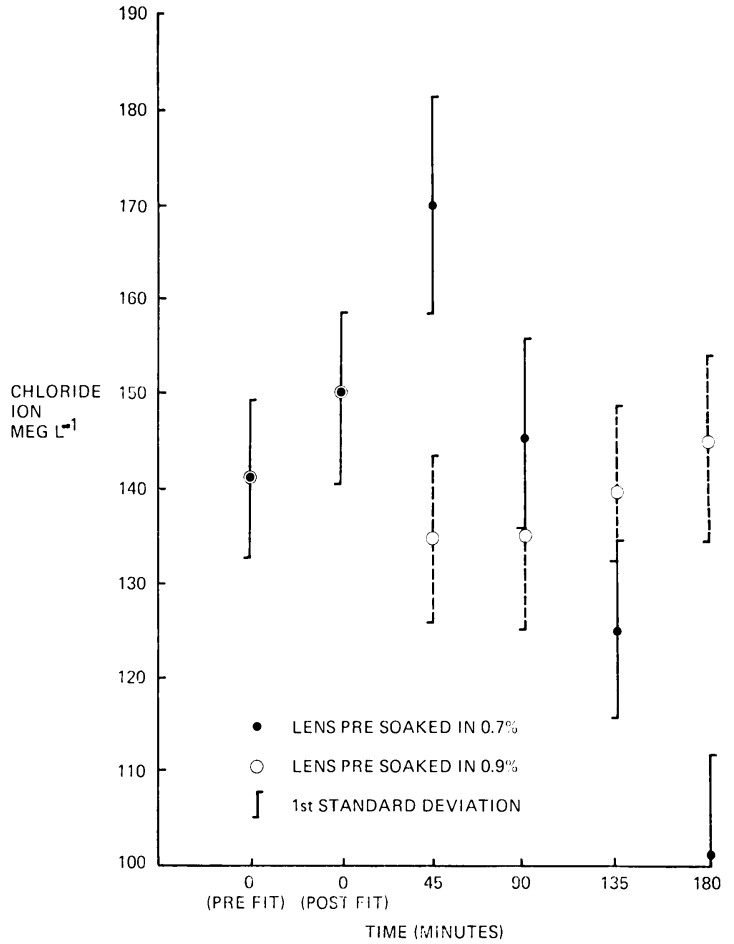

Fig. 5 Tear chloride ion levels

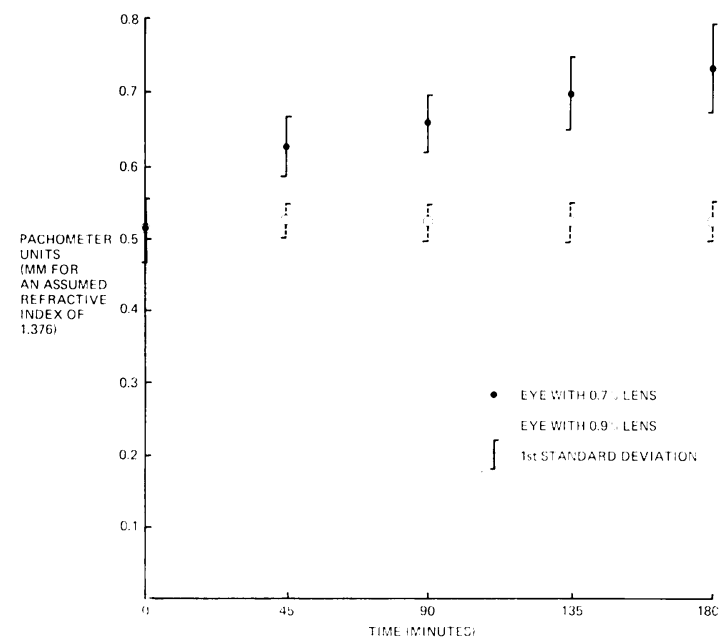

Fig. 6 Human corneal thickness as indicated by pachometry (central cornea)

the cornea, possibly owing to a greater interchange of tear fluid in this area.

Although caution is desirable in ascribing causal relationships to isolated phenomena under study, it is probable that the change in observed corneal thickness is directly attributable to the imbalance in

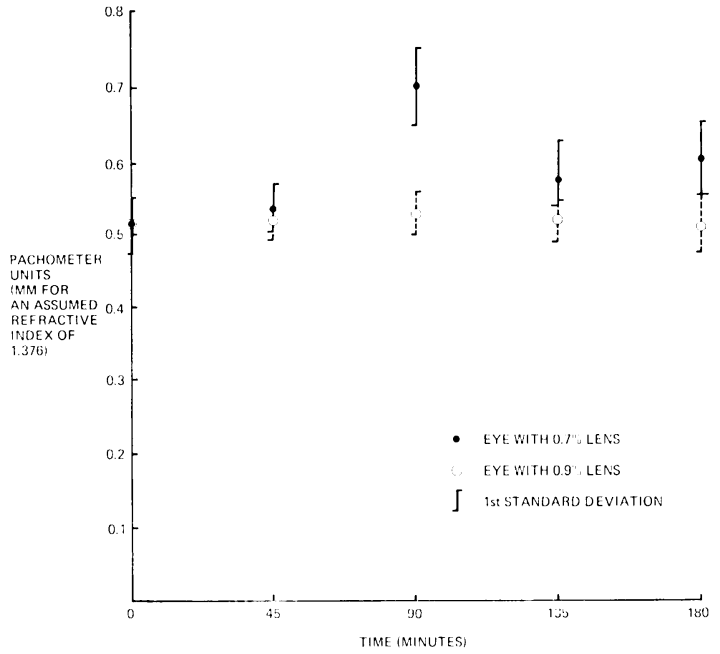

Fig. 7 Human corneal thickness as shown by pachometry ( $30^{\circ}$ superior region)

the tonicity of the presoaked soft lens. At present the longer-term clinical implications of these corneal changes are not understood. It would seem unlikely that the clinical response is as severe as the initial animal work would suggest. It is also possible that the fitting relationship influences the rate of interchange of tear fluid trapped between the contact lens and the cornea, and the severity of response may therefore be related to the mode of fit. A review of the literature reveals few instances of reported adverse response to soft lens wear, though Mackie (1977) has described a patient with corneal changes which would seem to fit into this particular pattern. It is probably wise for the clinician to emphasise to his patients the desirability of getting the correct composition for the solution. Possibly also the practice of patients preparing the saline should be reviewed.

The writers are indebted to Messrs Bausch and Lomb for financial support and assistance in the conduct of this research. They are also indebted to Dr R. Barry for preparation and photography of the histological specimens.

\section{References}

Boyd, J. R. (1975). Contact Lens Congress, Kansas City, February.

Kempster, A. J., Larke, J. R., and Marsters, J. (1975). The effect of hypertonic saline on human corneal hydiation. British Journal of Physiological Optics, 30, 16-19.

Mackie, I. (1977). British Contact Lens Association Conference, Torquay, April.

Mishima, S., and Hedbys, B. (1968). Measurement of corneal thickness by the Haag Streit pachometer, Archives of Ophthalmology, 80, 710-713. 